\title{
VDI
}

\section{REIHE 21}

ELEKTROTECHNIK

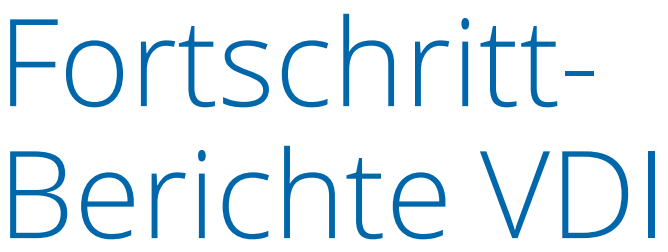

M. Sc. Manuel Oberneder, Büchlberg

NR. 424

Modellbildung,

Dimensionierung und modellbasierte

Regelung des Modularen

Mehrpunktstromrichters

zur systemoptimalen

Betriebsführung von

Drehstrommaschinen

BAND

$1 \mid 1$

VOLUME

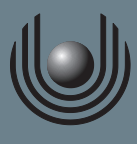

FernUniversität in Hagen

Schriften zur Informations-

https://doi.org/10.51202/9783186424211-1

und Kommunikationstechnik 


\title{
Modellbildung, Dimensionierung und modellbasierte Regelung des Modularen Mehrpunktstromrichters zur systemoptimalen Betriebsführung von Drehstrommaschinen
}

\author{
Dissertation zur Erlangung des akademischen Grades \\ eines Doktors der Ingenieurwissenschaften (Dr.-Ing.) \\ in der Fakultät Mathematik und Informatik \\ der FernUniversität in Hagen
}

vorgelegt von

M.Sc. Manuel Oberneder

geboren am 03.03.1987 in Hutthurm

Hagen, 2022 
1. Gutachter: Prof. Dr.-Ing. Detlev Hackstein, FernUniversität in Hagen

2. Gutachter: Prof. Dr.-Ing. Michael Saller, OTH Regensburg

Tag der mündlichen Prüfung 26.01.2022 


\section{VDI}

REIHE 21

ELEKTROTECHNIK
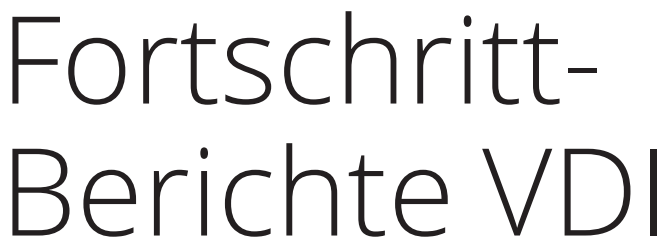

M. Sc. Manuel Oberneder, Büchlberg

NR. 424

Modellbildung,

Dimensionierung und modellbasierte

Regelung des Modularen

Mehrpunktstromrichters

zur systemoptimalen

Betriebsführung von

Drehstrommaschinen

BAND

1|1

VOLUME 


\section{VDI verlag}

Oberneder, Manuel

\section{Modellbildung, Dimensionierung und modellbasierte Regelung des Modularen Mehrpunktstromrichters zur systemoptimalen Betriebsführung von Drehstrommaschinen}

Fortschritt-Berichte VDI, Reihe 21, Nr. 424. Düsseldorf: VDI Verlag 2022.

204 Seiten, 119 Bilder, 11 Tabellen.

ISBN 978-3-18-342421-4, E-ISBN 978-3-18-642421-1, ISSN 0178-9481

71,00 EUR/VDI-Mitgliederpreis: 63,90 EUR

Für die Dokumentation: Modularer Mehrpunktstromrichter - Modelbasierte Regelung - Analytische Lösung Drehstrommaschine - Windenergieerzeugung - Auslegungsverfahren

Keywords: Modular Multilevel Converter - model-based control - analytical solution - three-phase electrical machine - wind power generation - design procedure

Die vorliegende Arbeit wendet sich an Ingenieur*innen und Wissenschaftler*innen aus der Elektrotechnik. Sie befasst sich mit der Ansteuerung von Drehstrommaschinen durch den Modularen Mehrpunktstromrichter (M2C) auf Basis einer allgemeinen Lastdefinition und der umfänglichen analytischen Lösung der Systemgleichungen. Diese Lösung bildet die Grundlage zur Regelung der Zweigenergien bei einer minimalen Komponentenbelastung, indem die Steuergrößen identifiziert und die Möglichkeiten einer modellbasierten Regelung aufgezeigt werden. Des Weiteren wird ein Auslegungsverfahren vorgestellt, welches mit geringer Rechenzeit eine umfängliche Variation der Systemparameter des M2Cs in Kombination mit einer Drehstrommaschine erlaubt. Die gezeigten Regelungs- und Ansteueralgorithmen werden außerdem mittels Simulationen sowie durch einen Versuchsaufbau verifiziert.

\section{Bibliographische Information der Deutschen Bibliothek}

Die Deutsche Bibliothek verzeichnet diese Publikation in der Deutschen Nationalbibliographie; detaillierte bibliographische Daten sind im Internet unter www.dnb.de abrufbar.

Bibliographic information published by the Deutsche Bibliothek (German National Library)

The Deutsche Bibliothek lists this publication in the Deutsche Nationalbibliographie

(German National Bibliography); detailed bibliographic data is available via Internet at www.dnb.de.

Schriften zur Informations- und Kommunikationstechnik Herausgeber:

Wolfgang A. Halang, Lehrstuhl für Informationstechnik Herwig Unger, Lehrstuhl für Kommunikationstechnik FernUniversität in Hagen

VDI Verlag GmbH | Düsseldorf 2022

Als Manuskript gedruckt. Printed in Germany. Vertrieb und Nachdruck VDI Verlag GmbH. ISBN 978-3-18-342421-4, E-ISBN 978-3-18-642421-1, ISSN 0178-9481

Das Werk steht unter Creative Commons Lizenz Namensnennung - Weitergabe unter gleichen Bedingungen 4.0 International (CC BY-SA 4.0)

\section{(i) (2)}




\section{Vorwort}

Die vorliegende Arbeit entstand parallel zu meiner beruflichen Tätigkeit als Abteilungsleiter bei der Siemens AG in der Entwicklung für Windkraftgeneratoren.

Bei Herrn Prof. Dr.-Ing. Detlef Hackstein bedanke ich mich sehr für das stets entgegengebrachte Interesse und die vertrauensvolle Zusammenarbeit sowie die Übernahme des Gutachtens.

Herrn Prof. Dr.-Ing. Michael Saller möchte ich ganz besonders für die jahrelange Zusammenarbeit und die große Unterstützung danken. Das entgegengebrachte Vertrauen und die vielen wertvollen Hinweise waren eine große Stütze. Sein Wirken war und ist für mich eine große Inspiration und der Grund für meinen stetigen Drang nach einem umfassenden Verständnis technischer Zusammenhänge.

Michael Scharnagl möchte ich an dieser Stelle für die hilfreichen Diskussionen und Anregungen danken. Außerdem gilt mein großer Dank den Kollegen der Siemens AG, die mich durch technische Diskussionen, beim Aufbau des Prüfstandes und durch die Bereitstellung von Hardware und Messmitteln tatkräftig unterstützt haben.

Meinen Eltern Gertraud und Ludwig sowie meinem Bruder Alexander danke ich für die familiäre Unterstützung, den stetigen Rückhalt und die Freiheiten die ich hatte um meinen Weg zu gehen.

Ein ganz besonderer Dank gilt letztendlich meiner Ehefrau Michaela. Durch ihre großartige Unterstützung und ihre unendliche Geduld hat sie wesentlich zum Gelingen dieser Arbeit beigetragen. Unser Sohn Jonas kann stolz sein, eine solche Mutter zu haben.

Hagen, im Januar 2022

Manuel Oberneder 


\section{Inhaltsverzeichnis}

Vorwort III

$\begin{array}{lll}\text { Symbolverzeichnis } & \text { VII }\end{array}$

$\begin{array}{lll}\text { Kurzfassung XVIII } & \text { XV }\end{array}$

$\begin{array}{ll}\text { Abstract } & \mathbf{X X}\end{array}$

1 Einleitung $\quad 1$

1.1 Motivation . . . . . . . . . . . . . . . . . 1

1.2 Eigenschaften und Klassifizierung von Mehrpunktstromrichtern . . 3

1.2.1 Neutral-Point Clamped Topologien . . . . . . . . . . . . 5

1.2.2 Flying Capacitor Topologie . . . . . . . . . . . . . . . 8

1.2.3 Cascaded H-Bridge Topologie . . . . . . . . . . . . . . 8

1.2.4 Hybrid Topologien . . . . . . . . . . . . . . . . . . . 9

1.2.5 Modular Multilevel Converter Topologie . . . . . . . . . . 10

2 Modellbildung 14

2.1 Modellbildung mit allgemeiner Lastdefinition . . . . . . . . . . . . 15

2.1.1 Allgemeines Differentialgleichungssystem . . . . . . . . . . 17

2.1.2 Definition der Zweigstrom- und Gleichtaktspannungsfunk-

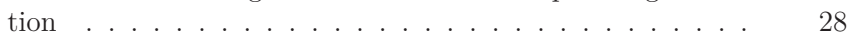

2.1.3 Analyse der symmetrischen Zweigenergien . . . . . . . . . . . 37

2.1.4 Analyse der asymmetrischen Zweigenergien . . . . . . . . . . 51

2.1.5 Separation der Last- und Umrichterströme . . . . . . . . . 56

2.1.6 Anfangsbedingung der Kondensatorspannung . . . . . . . 58

2.2 Maschinenmodell am Beispiel der permanentmagneterregten Synchronmaschine ................... 61

3 Regelung und Ansteuerung des elektrischen Triebstrangs $\quad 69$

3.1 Systemregelung durch die Zweigströme . . . . . . . . . . . . 70

3.2 Separate Umrichter- und Lastregelung . . . . . . . . . . . . . . . 72

3.2.1 Regelung der internen Umrichterströme . . . . . . . . . 75

3.2.2 Zweigenergieregelung ................ 77

3.2.3 Maschinenregelung am Beispiel der PMSM . . . . . . . . . 79

3.3 Modulation und Symmetrierung der Submodulspannungen . . . . 83

3.3.1 Modulationsmethoden ................ 84 
3.3.2 Sortieralgorithmus zur Symmetrierung der Submodulspannungen . . . . . . . . . . . . . . . . . . . 91

3.4 Systemsimulation mit diskretem Umrichtermodell . . . . . . . . . 93

3.4.1 Regelung der Zweigströme mittels Fehlerfunktion . . . . . 94

3.4.2 Validierung der Systemgleichungen . . . . . . . . . . . . 97

3.4.3 Separierung der Umrichter- und Lastregelung . . . . . . . 99

4 Verfahren zur Systemauslegung 102

4.1 Bestimmung der Betriebscharakteristik des PMSGs . . . . . . . . 105

4.2 Auslegungsverfahren zum M2C mit Maschinenlast . . . . . . . . . . 109

4.2.1 Bestimmung der Zweiginduktivität . . . . . . . . . . . . 112

4.2.2 Dimensionierung der Submodulkondensatoren . . . . . . . 116

4.2.2.1 Identifikation auslegungsrelevanter Betriebspunkte ...................... 117

4.2.2.2 Reduktion der Spannungsschwankung durch die

4.2.2.3 Minimierung der Kondensatorspannungsbelastung durch Kreisströme . . . . . . . . . . . . . 122

4.2.2.4 Temperaturbelastung der Submodulkondensatoren ......................... 124

4.2.3 Charakterisierung der Leistungshalbleiter . . . . . . . . . . 127

4.2.3.1 Regressionsmethode . . . . . . . . . . . . 127

4.2.3.2 Interpolationsmethode . . . . . . . . . . . . 136

4.2.4 Dimensionierung der Leistungshalbleiter . . . . . . . . . . . . 137

4.2.5 Auslegung der Zweigdrossel . . . . . . . . . . . . . . 144

5 Messtechnische Validierung der Betriebsführung $\quad 156$

5.1 Signalverarbeitung . . . . . . . . . . . . . . . . . . . . . . . . . . . . . 158

5.2 Betrieb der Modellanlage . . . . . . . . . . . . . . . . . 159

5.2.1 Vorladung der Submodulkondensatoren . . . . . . . . . . . 160

5.2.2 Betrieb des LV-M2Cs an einer induktiven Last . . . . . . . 162

5.2.3 Betrieb des LV-M2Cs mit einer Asynchronmaschine . . . . 163

6 Fazit 170

6.1 Zusammenfassung . . . . . . . . . . . . . . . . . . . . . . . 170

6.2 Ausblick . . . . . . . . . . . . . . . . . . 172

$\begin{array}{ll}\text { Literaturverzeichnis } & 174\end{array}$ 


\section{Symbolverzeichnis}

\section{Grundzeichen}

\begin{tabular}{|c|c|}
\hline$\Delta \widetilde{w}$ & Zeitvariante Zweigenergieschwankung \\
\hline$\Delta W$ & Zeitinvariante Zweigenergieschwankung \\
\hline$\Delta w$ & Summe der Zeitvarianten und -invarianten Zweigenergieschwankung \\
\hline$\hat{I}$ & Konstanter Stromspitzenwert \\
\hline$\hat{U}$ & Konstanter Spannungsspitzenwert \\
\hline$\psi$ & Zeitvarianter magnetischer Fluss \\
\hline$\rho$ & Dichte \\
\hline$\widetilde{i}$ & Zeitvarianter Strom \\
\hline$\widetilde{p}$ & Zeitvariante Leistung \\
\hline$\widetilde{s}$ & Zeitvariante Schaltfunktion \\
\hline$\tilde{u}$ & Zeitvariante Spannung \\
\hline$A$ & Fläche \\
\hline$B$ & Magnetische Induktion \\
\hline$C$ & Kumulierte Zweigkapazität \\
\hline$f$ & Elektrische Frequenz \\
\hline$G$ & Übertragungsfunktion \\
\hline$H$ & Magnetische Feldstärke \\
\hline$I$ & Zeitinvarianter Strom \\
\hline$i$ & Summe des Zeitvarianten und -invarianten Stroms \\
\hline$K$ & Knotenpunkt \\
\hline$L$ & Zeitinvariante Induktivität \\
\hline$l$ & Länge \\
\hline$M$ & Drehmoment \\
\hline$n$ & Drehzahl der elektrischen Maschine \\
\hline$P$ & Zeitinvariante Leistung \\
\hline$p$ & Summe der Zeitvarianten und -invarianten Leistung \\
\hline$R$ & Zeitinvarianter Widerstand \\
\hline & Summe der Zeitvarianten und -invarianten Schaltfunktion \\
\hline
\end{tabular}


T Zeitkonstante, Periodendauer

$t \quad$ Zeit

U Zeitinvariante Spannung

$u \quad$ Summe der Zeitvarianten und -invarianten Spannung

$V \quad$ Volumen

w Summe der Zeitvarianten und -invarianten Energie

\section{Weitere Formelzeichen}

$\alpha \quad$ Wärmeübergangskoeffizient

$\beta \quad$ Lastwinkel zwischen Polradspannungs- und Stromvektor

$\Delta \psi_{\text {Lzs }} \quad$ Differentieller Fluss der Zweigdrossel

$\Delta \widetilde{w}_{\Delta} \quad$ Energiehub

$\Delta \widetilde{w}_{\mathrm{zs}, \max } \quad$ Maximum des kumulierten Wechselanteils der Zweigenergieschwankung

$\Delta \widetilde{w}_{\text {zs,min }} \quad$ Minimum des kumulierten Wechselanteils der Zweigenergieschwankung

$\Delta \widetilde{w}_{\mathrm{zs}} \quad$ Kumulierter Wechselanteil der Zweigenergieschwankung

$\Delta i_{\mathrm{zs}} \quad$ Differentieller Strom der Zweigdrossel

$\Delta W_{\mathrm{j}} \quad$ Zeitinvariante Terme der Zweigenergieschwankung

$\Delta w_{\mathrm{j}} \quad$ Zeitvariante Terme der Zweigenergieschwankung

$\Delta w_{\mathrm{zs}} \quad$ Kumulierte Zweigenergieschwankung

$\gamma \quad$ Koordinatentransformationswinkel

$\hat{I}_{\mathrm{a}} \quad$ Amplitudenwert des Laststroms

$\hat{I}_{\mathrm{k}} \quad$ Amplitudenwert des Kreisstroms

$\hat{U}_{\text {a }} \quad$ Amplitudenwert der Lastspannung

$\hat{U}_{\mathrm{SN}} \quad$ Amplitudenwert der Gleichtaktspannung

$\lambda \quad$ Wärmeleitfähigkeit

$\Lambda_{\mathrm{i}} \quad$ Thermischer Leitwert

$\mu \quad$ Magnetische Permeabilität

$\mu_{0} \quad$ Magnetische Permeabilität der Luft

$\mu_{\mathrm{Fe}} \quad$ Magnetische Permeabilität von Eisen

$\nu \quad$ Ordnungszahl des Kreisstroms

$\nu_{\mathrm{SN}} \quad$ Ordnungszahl der Gleichtaktspannung

$\omega \quad$ Kreisfrequenz

$\psi_{\mathrm{dq}} \quad$ Verketteter magnetischer Fluss

$\psi_{\mathrm{d}} \quad$ Magnetischer Fluss in d-Richtung

$\psi_{\mathrm{L}} \quad$ Magnetischer Fluss der Zweigdrossel

$\psi_{\mathrm{pm}} \quad$ Magnetischer Fluss des Permanentmagneten 


\begin{tabular}{|c|c|}
\hline$\psi_{\mathrm{q}}$ & Magnetischer Fluss in q-Richtung \\
\hline$\rho$ & Dichte \\
\hline $\tan \delta$ & Dielektrischer Verlustfaktor \\
\hline$\theta$ & Elektrischer Drehwinkel \\
\hline$\Theta_{\mathrm{a}}$ & Temperatur Kühlmedium \\
\hline$\Theta_{\mathrm{D}, \mathrm{c}}$ & Gehäusetemperatur bei der Diode \\
\hline$\Theta_{D, j}$ & Sperrschichttemperatur der Diode \\
\hline$\Theta_{\mathrm{h}}$ & Kühlkörpertemperatur \\
\hline$\Theta_{\mathrm{K}}$ & Maximale Temperatur des Kondensators \\
\hline$\Theta_{\mathrm{m}}$ & Magnetische Durchflutung \\
\hline$\Theta_{\mathrm{T}, \mathrm{c}}$ & Gehäusetemperatur beim Transistor \\
\hline$\Theta_{\mathrm{T}, \mathrm{j}}$ & Sperrschichttemperatur des Transistors \\
\hline$\varphi$ & Phasenverschiebungswinkel zwischen Laststrom und -spannung \\
\hline$\varphi_{\mathrm{k}}$ & Phasenverschiebungswinkel des Kreisstroms \\
\hline$\varphi_{\mathrm{SN}}$ & Phasenverschiebungswinkel der Gleichtaktspannung \\
\hline$\varphi_{\mathrm{s}}$ & Phasenverschiebungswinkel der Phasenspannung \\
\hline$\vartheta$ & Polradwinkel \\
\hline$\widetilde{i}_{\mathrm{as}}$ & Zeitvarianter Laststrom \\
\hline$\widetilde{i}_{\mathrm{ks} \nu}$ & Harmonischer zeitvarianter Kreisstrom \\
\hline$\tilde{i}_{\mathrm{ks}}$ & Zeitvarianter Kreisstrom \\
\hline$\widetilde{i}_{\mathrm{Kzs}}$ & Zeitvarianter Kondensatorstrom \\
\hline$\widetilde{i}_{\mathrm{Z}}$ & Zeitvarianter Zwischenkreisstrom \\
\hline$\widetilde{p}_{\text {zS }}$ & Kumulierte zeitvariante Zweigleistung \\
\hline$\tilde{u}_{\text {as }}$ & Zeitvariante Lastspannung \\
\hline$\widetilde{u}_{\mathrm{Kzs}}$ & Kumulierte zeitvariante Zweigkondensatorspannung \\
\hline$\widetilde{u}_{\mathrm{SN}}$ & Zeitvariante Gleichtaktspannung \\
\hline$\widetilde{u}_{\mathrm{zs}}$ & Kumulierte zeitvariante Zweigspannung \\
\hline$\widetilde{u}_{\mathrm{zZ}}$ & Zeitvariante Zwischenkreisspannung \\
\hline$\xi$ & Wicklungsfaktor \\
\hline$\zeta_{\mathrm{L}}$ & Windungszahl der Zweigdrossel \\
\hline$A_{\mathrm{Fe}}$ & Querschnittsfläche des Eisenkerns \\
\hline$c$ & spezifische Wärmekapazität \\
\hline$C_{\mathrm{i}}$ & Wärmekapazität \\
\hline$C_{\mathrm{K}}$ & Kapazität des einzelnen Kondensators \\
\hline$C_{\mathrm{SM}}$ & Submodulkapazität \\
\hline$F_{\text {rel }}$ & Relativer Regressionsfehler \\
\hline$H_{1}$ & Magnetische Feldstärke des umwickelten Schenkels \\
\hline $\mathrm{H}_{2}$ & Magnetische Feldstärke des äußeren Schenkels \\
\hline
\end{tabular}


$H_{3} \quad$ Magnetische Feldstärke des Jochs

$H_{\delta} \quad$ Magnetische Feldstärke des Luftspalts

$I_{\text {as }} \quad$ Zeitinvarianter Laststrom

$I_{\mathrm{C}} \quad$ Kollektorstrom des Transistors

$i_{\mathrm{C}} \quad$ Zeitabhängiger Kollektorstrom

$I_{\mathrm{F}} \quad$ Diodenstrom

$i_{\mathrm{F}} \quad$ Zeitabhängiger Diodenstrom

$I_{\mathrm{ks}} \quad$ Zeitinvarianter Kreisstrom

$i_{\mathrm{ks}} \quad$ Kreisstrom

$I_{\mathrm{Kzs}} \quad$ Zeitinvarianter Kondensatorstrom

$i_{\mathrm{Kzs}} \quad$ Zweigkondensatorstrom

$i_{\text {Lzs }} \quad$ Strom durch die Zweigdrossel

$i_{\mathrm{zs}} \quad$ Zweigstrom

$I_{Z} \quad$ Zeitinvarianter Zwischenkreisstrom

$k \quad$ Zähloperator

$k_{\text {Mod }} \quad$ Modulationsgrad

$K_{\mathrm{S}} \quad$ Faktor der Fehlerverstärkung

$K_{\mathrm{uD}} \quad$ Exponent des Spannungsverhältnisses der Ausschaltverluste der Diode

$K_{\mathrm{uT}} \quad$ Exponent des Spannungsverhältnisses der Ein- und Ausschaltverluste des Transistors

L Zweiginduktivität

$l_{\delta} \quad$ Länge des Luftspalts

$L_{\mathrm{dd}}, L_{\mathrm{dd}} \quad$ Differentielle Induktivitäten in der d- und q-Achse

$L_{\mathrm{dq}}, L_{\mathrm{qd}} \quad$ Differentielle Induktivitäten zwischen d- und q-Achse

$l_{\mathrm{H}}$

Horizontale Länge des Eisenkerns

$L_{\mathrm{L}} \quad$ Differentielle Induktivität der Zweigdrossel

$L_{\max } \quad$ Maximale Zweiginduktivität

$L_{\mathrm{pn}}, L_{\mathrm{np}} \quad$ Differentielle Koppelinduktivitäten der Zweigdrossel

$L_{\mathrm{pp}}, L_{\mathrm{nn}} \quad$ Differentielle Hauptinduktivitäten der Zweigdrossel

$l_{\mathrm{V}}$

Vertikale Länge des Eisenkerns

$L_{\mathrm{zZ}}$

$L_{\mathrm{Z}}$

Zwischenkreisinduktivität des positiven oder negativen Zweiges

$M_{\delta} \quad$ Luftspaltmoment

$m_{\text {off }} \quad$ Zähler der Ausschaltpulse der Diode

$M_{\text {on }} \quad$ Anzahl der Ausschaltpulse der Diode

$M_{\mathrm{v}} \quad$ Verlustdrehmoment

$n_{\mathrm{m}} \quad$ Mechanische Drehzahl

$N_{\text {off }} \quad$ Anzahl der Ausschaltpulse des Transistors 


\begin{tabular}{|c|c|}
\hline$n_{\text {off }}$ & Zähler der Ausschaltpulse des Transistors \\
\hline$N_{\text {on }}$ & Anzahl der Einschaltpulse des Transistors \\
\hline$n_{\text {on }}$ & Zähler der Einschaltpulse des Transistors \\
\hline$P_{\mathrm{D}, \text { cond }}$ & Durchlassverluste der Diode \\
\hline$P_{\mathrm{D}, \text { off }}$ & Ausschaltverluste der Diode \\
\hline$P_{\mathrm{D}}$ & Gesamtverluste der Diode \\
\hline$P_{\mathrm{j}}$ & Zeitinvariante Terme der Zweigleistung \\
\hline$p_{\mathrm{j}}$ & Zeitvariante Terme der Zweigleistung \\
\hline$P_{\mathrm{T}, \mathrm{cond}}$ & Durchlassverluste des Transistors \\
\hline$P_{\mathrm{T}, \mathrm{off}}$ & Ausschaltverluste des Transistors \\
\hline$P_{\mathrm{T}, \mathrm{on}}$ & Einschaltverluste des Transistors \\
\hline$P_{\mathrm{T}}$ & Gesamtverluste des Transistors \\
\hline$P_{\mathrm{v}, \mathrm{D}}$ & Dielektrische Verlustleistung des Kondensators \\
\hline$P_{\mathrm{v}, \mathrm{K}}$ & Kondensatorverlustleistung \\
\hline$P_{\mathrm{v}, \mathrm{pm}}$ & Magnetverlustleistung \\
\hline$P_{\mathrm{v}, \mathrm{R}}$ & Ohmsche Verlustleistung des Kondensators \\
\hline$P_{\mathrm{v}, \mathrm{r}}$ & Reibungs- und Ventilationsverlustleistung \\
\hline$P_{\mathrm{zs}}$ & Kumulierte zeitinvariante Zweigleistung \\
\hline$p_{\mathrm{zs}}$ & Kumulierte Zweigleistung \\
\hline$P_{\mathrm{Z}}$ & Zwischenkreisleistung \\
\hline$R$ & Zweigwiderstand \\
\hline$R_{\mathrm{ESR}}$ & Äquivalenter Serienersatzwiderstand des Kondensators \\
\hline$R_{\text {ha }}$ & Thermischer Widerstand Kühlkörper zu Kühlmedium \\
\hline$R_{\mathrm{i}, \alpha}$ & Wärmeübergangswiderstand \\
\hline$R_{\mathrm{i}, \lambda}$ & Wärmeleitwiderstand \\
\hline$R_{\mathrm{i}}$ & Thermischer Widerstand \\
\hline$R_{\mathrm{m}, 1}$ & Magnetischer Widerstand des umwickelten Schenkels \\
\hline$R_{\mathrm{m}, 2}$ & Magnetischer Widerstand des äußeren Schenkels \\
\hline$R_{\mathrm{m}, 3}$ & Magnetischer Widerstand des Jochs \\
\hline$R_{\mathrm{m}, \delta}$ & Magnetischer Widerstand des Luftspalts \\
\hline$R_{\mathrm{m}}$ & Magnetischer Widerstand \\
\hline$R_{\mathrm{S}}$ & Serienersatzwiderstand des Kondensators \\
\hline$R_{\mathrm{T}, \mathrm{ch}}$ & Thermischer Widerstand Gehäuse zu Kühlkörper bei der Diode \\
\hline$R_{\mathrm{T}, \mathrm{ch}}$ & Thermischer Widerstand Gehäuse zu Kühlkörper beim Transistor \\
\hline$R_{\mathrm{T}, \mathrm{jc}}$ & Thermischer Widerstand Sperrschicht zu Gehäuse bei der Diode \\
\hline$R_{\mathrm{T}, \mathrm{jc}}$ & Thermischer Widerstand Sperrschicht zu Gehäuse beim Transistor \\
\hline$R_{\mathrm{th}}$ & Thermischer Widerstand des Kondensators \\
\hline$R_{\mathrm{zZ}}$ & Zwischenkreiswiderstand des positiven oder negativen Zweiges \\
\hline
\end{tabular}


$R_{Z}$

$S_{\mathrm{CE}}$

$S_{\mathrm{F}}$

$S_{\text {off }}$

$S_{\text {on }}$

$S_{\text {rec }}$

$s_{\mathrm{zS}}$

$t_{0}$

$T_{\text {PWM }}$

$U_{0}$

$U_{\Delta}$

$U_{\text {as }}$

$U_{\mathrm{CE}, \mathrm{D}}$

$U_{\mathrm{CE}, \text { Interp }}$

$U_{\mathrm{CE}}$

$u_{\mathrm{C}}$

$u_{\mathrm{Ds}}$

$U_{\mathrm{F}, \mathrm{D}}$

$U_{\mathrm{F}, \text { Interp }}$

$U_{\mathrm{F}}$

$u_{\mathrm{F}}$

$U_{\mathrm{K}, \mathrm{N}}$

$u_{\mathrm{K}, \mathrm{SM}}$

$U_{\mathrm{Kzs}}$

$u_{\mathrm{Kzs}}$

$u_{\text {Lzs }}$

$u_{\mathrm{LzZ}}$

$U_{\text {pn }}$

$u_{\mathrm{Rzs}}$

$u_{\mathrm{RzZ}}$

$U_{\mathrm{r}}$

$U_{\mathrm{SN}}$

$U_{\mathrm{Z}, \min }$

$u_{\mathrm{zs}, \min }$

$U_{\mathrm{zs}}$

$u_{\mathrm{zs}}$

$U_{\mathrm{zZ}}$

$W_{\text {off,D }}$

Gesamtwert der Zwischenkreiswiderstände

Approximationsfehler der Kollektor-Emitter-Spannung

Approximationsfehler der Diodendurchlassspannung

Approximationsfehler der Transistorausschaltenergie

Approximationsfehler der Transistoreinschaltenergie

Approximationsfehler der Diodenausschaltenergie

Schaltfunktion des Ersatzzweigmoduls

Anfangszeitpunkt

Zeitdauer einer PWM-Periode

Konstanter Spannungsabfall

Kondensatorrippelspannung

Zeitinvariante Lastspannung

Datenpunkte der Kollektor-Emitter-Spannung

Interpolierte Kennlinie der Kollektor-Emitter-Spannung

Kollektor-Emitter-Spannung

Zeitabhängige Kollektor-Emitter-Spannung

Spannungsabfall Zweiginduktivität und -widerstand

Datenpunkte der Durchlasskennlinie der Diode

Interpolierte Durchlasskennlinie der Diode

Durchlassspannung der Diode

Zeitabhängige Diodenspannung

Kondensatornennspannung

Kondensatorspannung des Submoduls

Kumulierte zeitinvariante Zweigkondensatorspannung

Kumulierte Zweigkondensatorspannung

Spannungsabfall an der Zweiginduktivität

Spannungsabfall an der Zwischenkreisinduktivität

Gesamtspannung des Zwischenkreises

Spannungsabfall am Zweigwiderstand

Spannungsabfall am Zwischenkreiswiderstand

Konstante Spannungsreserve

Zeitinvariante Gleichtaktspannung

Minimale Zwischenkreisspannung

Minimale Zweigspannung

Kumulierte zeitinvariante Zweigspannung

Kumulierte Zweigspannung

Zeitinvariante Zwischenkreisspannung des jeweiligen Zweiges

Datenpunkte der Ausschaltenergie des Transistors 


$\begin{array}{ll}W_{\text {off,Interp }} & \text { Interpolierte Kennlinie Ausschaltenergie des Transistors } \\ W_{\text {off }} & \text { Ausschaltenergie des Transistors } \\ W_{\text {on,D }} & \text { Datenpunkte der Einschaltenergie des Transistors } \\ W_{\text {on,Interp }} & \text { Interpolierte Kennlinie der Einschaltenergie des Transistors } \\ W_{\text {on }} & \text { Einschaltenergie des Transistors } \\ W_{\text {rec,D }} & \text { Datenpunkte der Ausschaltenergie der Diode } \\ W_{\text {rec,Interp }} & \text { Interpolierte Kennlinie der Ausschaltenergie der Diode } \\ W_{\text {rec }} & \text { Ausschaltenergie der Diode } \\ w_{\text {zs }} & \text { Kumulierte Zweigenergie } \\ Z_{\mathrm{p}} & \text { Polpaarzahl } \\ Z_{\mathrm{SM}} & \text { Anzahl der Submodule je Zweig }\end{array}$

\section{Abkürzungen}

$\begin{array}{ll}\text { AC } & \text { Alternating Current } \\ \text { AMP } & \text { Strommessplatine } \\ \text { ANLM } & \text { Averaging Nearest Level Modulation } \\ \text { ANPCML } & \text { Active Neutral Point Clamped Multilevel } \\ \text { ANPC } & \text { Active Neutral Point Clamped } \\ \text { APOC } & \text { Alternative Phase Opposition Carrier } \\ \text { ARM } & \text { Advanced RISC Machines } \\ \text { ASNPC } & \text { Active Stacked Neutral Point Clamped } \\ \text { CAN } & \text { Controller Area Network } \\ \text { CHB } & \text { Cascaded H-Bridge } \\ \text { CM } & \text { Common Mode } \\ \text { CSI } & \text { Current Source Inverter } \\ \text { DC } & \text { Direct Current } \\ \text { DD } & \text { Direct Drive } \\ \text { DFIG } & \text { Doubly Fed Induction Generator } \\ \text { DM } & \text { Differential Mode } \\ \text { DUT } & \text { Device Under Test } \\ \text { EESG } & \text { Electrically Excited Synchronous Generator } \\ \text { eM } & \text { Elektrische Maschine } \\ \text { FFT } & \text { Fast Fourier Transform } \\ \text { FL-LSC } & \text { Full Load Line Side Converter } \\ \text { FL-MSC } & \text { Full Load Machine Side Converter } \\ \text { FLC } & \text { Flying Capacitor } \\ \text { FL } & \text { Full Load } \\ & \end{array}$


FPGA Field-Programmable Gate Array

GD Geared Drive

GEN Generator

HGÜ Hochspannungs-Gleichstrom-Übertragung

HT Hybride Topologie

HVAC High Voltage Alternating Current

HVDC High Voltage Direct Current

IGBT Insulated Gate Bipolar Transistor

IGCT Integrated Gate-Commutated Thyristor

LSC Line Side Converter

Lsg Lösung

LV Low Voltage

LWL Lichtwellenleiter

M2C Modularer Mehrpunktstromrichter

MOSFET Metal Oxide Semiconductor Field-Effect Transistor

MPC Multi Point Clamped

MSC Machine Side Converter

MVAC Medium Voltage Alternating Current

NLM Nearest Level Modulation

NPC Neutral Point Clamped

NPP Neutral Point Piloted

NP Neutralpunkt

PC Personal Computer

PDC Phase Disposition Carrier

PEBB Power Electronic Building Block

$\mathrm{PH} \quad$ Phase

PL-LSC Part Load Line Side Converter

PL-MSC Part Load Machine Side Converter

PL Part Load

PMSG Permanent Magnet Synchronous Generator

PMSM Permanent Magnet Synchronous Machine

POC Phase Opposition Carrier

PSC Phase Shifted Carrier

PWM Pulsweitenmodulation

SCIG Squirrel Cage Induction Generator

SG Synchronous Generator

SMC Stacked Multi-Cell

SM Submodul 


$\begin{array}{ll}\text { SNPC } & \text { Stacked Neutral Point Clamped } \\ \text { SoC } & \text { System-on-a-Chip } \\ \text { SP } & \text { Sternpunkt } \\ \text { SÜ } & \text { Systemüberwachung } \\ \text { TCC } & \text { Transistor Clamped Converter } \\ \text { TSP } & \text { Treiberschnittstellenplatine } \\ \text { UART } & \text { Universal Asynchronous Receiver Transmitter } \\ \text { VMP } & \text { Spannungsmessplatine } \\ \text { VSC } & \text { Voltage Source Converter } \\ \text { WQN } & \text { Wärmequellennetz } \\ \text { ZS } & \text { Zentrale Steuerung }\end{array}$

\section{Matrizen}

$\begin{array}{ll}\boldsymbol{\Lambda} & \text { Matrix der Leitwerte } \\ \boldsymbol{\Psi} & \text { Matrix des magnetischen Flusses } \\ \boldsymbol{A} & \text { Matrix der Temperaturen } \\ \boldsymbol{B} & \text { Matrix der Kondensatorspannungsterme } \\ \boldsymbol{i}_{\mathrm{a}} & \text { Matrix der Zweigstrom- und Spannungsterme } \\ \boldsymbol{i} & \text { Matrix des Strangstroms der PMSM } \\ \boldsymbol{M}_{\boldsymbol{1}} & \text { Matrix der Zweigströme } \\ \boldsymbol{M}_{\mathbf{2}} & \text { Induktivitätsmatrix } \\ \boldsymbol{M}_{\mathbf{3}} & \text { Widerstandsmatrix } \\ \boldsymbol{M}_{\mathbf{4}} & \text { Faktormatrix } \\ \boldsymbol{M}_{\mathbf{5}} & \text { Induktivitätsmatrix der Schaltfunktionsgleichungen } \\ \boldsymbol{M}_{\mathbf{6}} & \text { Widerstandsmatrix der Schaltfunktionsgleichungen } \\ \boldsymbol{M}_{\boldsymbol{7}} & \text { Faktormatrix der Schaltfunktionsgleichungen } \\ \boldsymbol{M}_{\mathbf{8}} & \text { Induktivitätsmatrix der Kondensatorspannungsgleichungen } \\ \boldsymbol{M}_{\mathbf{9}} & \text { Widerstandsmatrix der Kondensatorspannungsgleichungen } \\ \boldsymbol{P} & \text { Faktormatrix der Kondensatorspannungsgleichungen } \\ \boldsymbol{s} & \text { Matrix der Verlustleistungen } \\ \boldsymbol{T}_{\mathrm{C}} & \text { Matrix der Schaltfunktionen } \\ \boldsymbol{U}_{\boldsymbol{\delta}} & \text { Clarke Transformationsmatrix } \\ \boldsymbol{U}_{\mathrm{a}} & \text { Matrix der Luftspaltspannung der PMSM } \\ \boldsymbol{u}_{\mathrm{K}} & \text { Matrix der Strangspannung der PMSM } \\ \boldsymbol{u} & \text { Matrix der Kondensatorspannungen } \\ & \text { Matrix der Last-, Zwischenkreis- und Gleichtaktspannungen }\end{array}$




\section{Indizes}

$*$

Poly, 2

Poly, 3

Pot

$\mathrm{p}$

q

ref

$\mathrm{R}$

$\mathrm{r}$

$r$

$\mathrm{s}+$

s-

Sollwert

Anfangswert oder -bedingung

Auf $\alpha$-Achse projizierte Größe

Auf $\beta$-Achse projizierte Größe

Umrechnungsexponent der Reibungs- und Ventilationsverluste

Luftspaltbezogene Größen

Ordnungszahl

Auf Magnetfluss bezogene Größe

Asymmetrisch

Lastgrößen

Kollektor-Emitter Größe

Auf d-Achse projizierte Größe

Auf Elektroblech bezogene Größe

Auf Feldschwächung bezogene Größe

Auf Diode bezogene Größe

Interne Umrichtergrößen

Zähler der Ergebnisterme

Kondensatorgrößen

Auf den Kreisstrom bezogene Größe

Linearer Ansatz

Induktive Größen

Zeitlich lineare Größen

Mechanische Größe

Negativer Zweig

Auf Permanent Magnet bezogene Größe

Polynomansatz zweiter Ordnung

Polynomansatz dritter Ordnung

Potenzfunktion

Positiver Zweig

Auf q-Achse projizierte Größe

Referenz

Ohmsche Widerstandsgrößen

Auf Reibung bezogene Größe

Reibung und Ventilation

Allgemeiner Platzhalter der nachfolgenden Phase

Allgemeiner Platzhalter der vorherigen Phase 
$\mathrm{SM}$

SN

$\mathrm{S}$

$\mathrm{s}$

$\mathrm{U}, \mathrm{V}, \mathrm{W}$

$\mathrm{V}$

W

Z

Z

Auf ein Submodul bezogene Größe

Gleichtaktgrößen

Symmetrisch

Allgemeiner Platzhalter für die Phasen U, V und W

Größen der jeweiligen Phase

Verlustterm

Zeitliche Wechselgrößen

Zwischenkreisgrößen

Allgemeiner Platzhalter für die Zweige $\mathrm{p}$ und $\mathrm{n}$ 


\section{Kurzfassung}

Die vorliegende Arbeit thematisiert die Modellbildung und darauf aufbauend die Betriebsführung und Dimensionierung des modularen Mehrpunktstromrichters (M2C) als auch die messtechnische Überprüfung des entwickelten Regelungsverfahrens an einem Prüfstand. Durch die hohe Modularität des M2Cs wird dieser vorrangig für die Hochspannungs-Gleichstrom-Übertragung praktisch eingesetzt. Trotz der Restriktionen beim Betrieb mit einer geringen Betriebsfrequenz wird dieser Umrichtertyp aber auch als Antriebsumrichter verwendet. Hier sind vor allem Anwendungen geeignet, welche ein ansteigendes Drehmoment bei gleichzeitiger Erhöhung der Drehzahl fordern. Dies ist beispielsweise für Pumpen- oder Lüfteranwendungen der Fall. Da dazu vor allem auch Windkraftturbinen zählen, welche durch den Energiewandel zunehmend an Bedeutung gewinnen, wird dieser Anwendungsfall in der vorliegenden Arbeit als Beispiel für die Systemdimensionierung angeführt.

Die Entwicklung und Analyse der Betriebsstrategien basiert auf einem tiefen Verständnis der Systemeigenschaften. Gegenstand dieser Arbeit ist es deshalb im ersten Schritt ein vollständiges Modell des M2Cs mit einer allgemeinen Lastdefinition zu erarbeiten, mit welchem eine analytische Lösung der Systemgleichungen für den stationären Fall möglich ist. Durch die analytische Ermittlung der einzelnen Lösungsterme wird eine Festlegung von zielspezifischen Auslegungskriterien sowie von optimalen Betriebsstrategien erreicht. Die allgemein verwendeten Formulierungen und die Darstellungen durch Einzelterme können weiterhin die Wirkmechanismen und deren Wechselwirkungen umfassend aufzeigen. Mittels der allgemeinen Lastdefinition wird des Weiteren die Möglichkeit aufgezeigt, ein sättigungsabhängiges Maschinenmodell auf Basis von Finite Elemente Simulationen in die Lösung der Gleichungen zu integrieren. Die Modellgleichungen erlauben ebenfalls eine einfache Vorausberechnung und damit die Optimierung der Spannungsaussteuerung der Zweige, woraus eine höhere Ausnutzung der Kondensatorspannung resultiert.

Auf Basis der analytischen Modellbildung wird im Weiteren ein Regelungsverfahren vorgestellt, welches modellbasiert die Eingangsgrößen des Energiereglers festlegt und damit eine hohe Stabilität erreicht. Die Trennung der Umrichter- und Lastregelung führt gleichzeitig zu einer entkoppelten und dynamischen Ansteuerung der Drehstrommaschine. Es handelt sich dabei um eine kaskadierte Regelungsstruktur durch welche höherfrequente Kreisstromformen sowie die Gleichtaktspannung vorgegeben werden können, um eine Minimierung der Kondensatorspannungsbelastung zu erreichen. Die optimierten Zweigströme und die entsprechende Gleichtaktspannung können durch das allgemeine Umrichtermodell vorab ermittelt werden.

Die Anwendung der Modellgleichungen wird folglich am Beispiel des elektrischen Triebstrangs für eine Windkraftanlage gezeigt und gleichzeitig darauf hingewiesen, 
welche Systemoptimierungen möglich sind, wenn die elektrische Drehstrommaschine in den Parameterraum miteinbezogen wird. Im Gegensatz zur numerischen Lösung der Gleichungssysteme bieten die analytischen Lösungen den Vorteil einer geringeren Rechenzeit, wodurch eine aufwendige Systemuntersuchung über den gesamten Betriebsbereich möglich wird. Damit werden die Einzelkomponenten des M2Cs, welche sich aus den Halbleitern, den Kondensatoren und Zweigdrosseln zusammensetzen, möglichst hoch ausgenutzt, um den Komponentenaufwand gering zu halten. Auf Basis von Finite Elemente Simulationen wird die Zweigdrossel im Detail betrachtet, um die Einflüsse von ge- bzw. entkoppelten Zweigdrosseln sowie der entsprechenden Wickelschemata aufzuzeigen.

Die Implementierung der Regelungs- und Ansteueralgorithmen in einer Niederspannungsmodellanlage des M2Cs belegt im Weiteren die Gültigkeit der aufgezeigten Modellgleichungen. Außerdem wird die Funktion der Regelungs- und Ansteueralgorithmen für verschiedene Betriebszustände sowohl mit einer Lastdrossel als auch einer Asynchronmaschine validiert. 


\section{Abstract}

This thesis deals with the modeling, and based on that, with the control and design of the Modular Multilevel Converter (M2C) as well as the validation of the developed control concept on a test bench. Because of the high modularity, the $\mathrm{M} 2 \mathrm{C}$ is mainly used for High-Voltage-Direct-Current applications. Despite the restrictions on low frequency operation, the $\mathrm{M} 2 \mathrm{C}$ is used for electrical drive systems as well. For drive applications this converter type is especially suitable for loads with simultaneously increasing torque and speed characteristics. This is e.g. the case for pump and fan applications. Wind turbines, which are gaining popularity due to the challenges of the climate change, are having these characteristics too. Therefore, this application is used as an example for the proposed design strategy.

The development and analysis of the operation strategies are based on a fundamental insight in the system behavior. This is the reason why this thesis deals firstly with a full model of the $\mathrm{M} 2 \mathrm{C}$ with a generally defined load model which allows for an analytical solution of the system equations for the stationary case. The determination of the respective system terms makes target orientated design criterias and optimized operation strategies possible. Furthermore, the usage of respective expressions leads to a detailed insight in the effects and interaction of the corresponding mechanisms. With the general load definition, it's possible to integrate a saturation model of the electrical machine into the system equations. In addition, the system model gives the possibility of optimizing the arm voltages, which results in highly utilized arm capacitors.

Based on the analytical model, a control scheme is demonstrated which gets the input parameters for the energy controller from the system equations and therefore allows for a high dynamic control of the electric machine. Separating the converter and load control gives the opportunity for a decoupled and dynamic control of the electric machine. This is based on a cascaded control scheme which uses circulating current harmonics and the common mode voltage as an input to minimize voltage ripples on the submodule capacitors. The optimized arm currents and the common mode voltage are determined by the general system model upfront.

The application of the system equations is shown on the electrical drivetrain of a wind turbine to demonstrate the possibility of system optimizations by considering the electrical machine in the parameter space. In contrast to a numerical simulation, the analytical approach gives the advantage of saving time and allows consequently for a general investigation on the system level over the entire operating range. With this the specific components of the $\mathrm{M} 2 \mathrm{C}$, which are the semiconductors, the capacitors and the arm inductors, are highly utilized to save costly components. Following, the arm inductors are calculated based on finite element simulations which shows 
the impact of coupled and decoupled arm inductors and various winding schemes. The implementation of the control and drive algorithms on a low voltage $\mathrm{M} 2 \mathrm{C}$ prototype proves the demonstrated solution of the system equations. Despite that the control and drive algorithms are tested for various operation scenarios with an inductive load and an asynchronous machine. 
